\title{
Modeling Arterial Wave Reflection with Difference Equations
}

\author{
Laura Lotteraner $^{1 *}, 2$, Bernhard Hametner ${ }^{2}$, Siegfried Wassertheurer ${ }^{2}$ \\ ${ }^{1}$ Institute of Analysis and Scientific Computing, TU Wien, Wiedner Hauptstraße 8-10, \\ 1040 Vienna, Austria; *Iaura.lotteraner@tuwien.ac.at \\ ${ }^{2}$ Center for Health \& Bioresources, AIT Austrian Institute of Technology, Giefinggasse 4, \\ 1210 Vienna, Austria
}

SNE 28(4), 2018, 157-164, DOI: 10.11128/sne.28.tn.10443

Received: September 20, 2018; Revised November 14, 2018;

Accepted: November 20, 2018

SNE - Simulation Notes Europe, ARGESIM Publisher Vienna,

ISSN Print 2305-9974, Online 2306-0271, www.sne-journal.org

Abstract. A simple difference equation model is presented that describes the development of blood pressure in the ascending aorta over time. Reflections both at one and more sites in the arterial system as well as re-reflections of reflected waves at the heart are considered. Model parameters include reflection magnitude, pulse wave velocity, reflection site positions and heart rate. The model is discussed both analytically and numerically by implementation in MATLAB. Comparison of blood pressure curves generated by the model and blood pressure curves measured in patients demonstrates that the model is able to represent reality to some extent. Results can be improved by adding reflection sites which warrants further research.

\section{Introduction}

Hypertension (elevated blood pressure) is a major cause of death mainly but not only in developed countries. According to the World Health Organization [1] hypertension accounts for about $12.8 \%$ of the annual deaths worldwide. In order to improve diagnosis and prevention it is crucial to understand the underlying dynamics in the cardiovascular system.

Mathematical models can help with this. In different types of models, many aspects of blood pressure have been considered by previous researchers. Traditional models are often based on fluid dynamics [2, 3] or use the analogy between the cardiovascular system and an electric circuit [4]. New approaches, however, are possible.
A large number of models involve the description of wave reflections [5], a phenomenon that occurs when blood pressure waves coming from the heart (forward waves) arrive at parts of the arterial system where vessel properties change, such as bifurcations. The forward waves are reflected and produce backward waves. This idea has been further extended by researchers $[6,7]$ who argued that backward waves are re-reflected at the heart. However, only few models exist that address this issue and it is poorly understood so far.

In this paper a simple difference equation model for aortic blood pressure is presented that focuses on the characterization of pulse wave reflections and rereflections. The properties of the model, such as the influence of various parameters on the results, are discussed.

\section{The Model}

Model Design. Even though blood pressure dynamics are time-continuous, time-discrete difference equations are a valuable tool to describe reflection effects from a finite number of reflection sites. Blood pressure at a fixed point in time and space can be calculated as the sum of blood pressure values at previous times, determined by the choice of reflection sites.

The arterial system is considered a single uniform tube with the heart, connected to the system via the aortic valve (AV), on one end and an elastic closed boundary, referred to as (single) distal reflection site (RS), on the other, see Figure 1. This idea has been applied in many different models and has been proved to be a reasonable approach by different authors [8]. It has even been used to model repeated reflections in the arterial system [7]. A measuring site (MS) is fixed in the ascending aorta, close to the aortic valve. This so-called aortic root is of particular interest because it allows to 
assess the interaction of reflections in the vascular system and the heart [9].

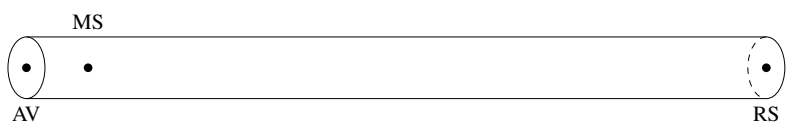

Figure 1: Tube model of the arterial system as presented above. AV - aortic valve, MS - measuring site, RS distal reflection site.

The distance between measuring site and distal reflection site will be named effective length in this paper, a terminology that expresses the reduction of multiple reflection effects to one. Analogously, the distal reflection site is also named effective reflection site. Often $[8,10]$ the aortic bifurcation is mentioned as the principal bifurcation site, the physiological counterpart of the effective reflection site.

Previous tube models have considered at least one spatial dimension and take into account the elastic properties of the tube wall [11]. The model presented in this paper, however, is dimensionless, only depending on time. It describes the development of blood pressure $P$ at the fixed measuring site over time. Therefore all spatial information has to be transformed into temporal information.

Model Parameters. The parameters of the model describe both the heart and the arterial system.

Reflections are described by real-valued reflection coefficients, $0<R_{D}<1$ for the distal reflection site and $0 \leq R^{a v} \leq 1$ for the aortic valve. The reflection coefficients include information on the elasticity properties of the arterial bed and the damping of pulse waves as they propagate through the blood [12]. While $R_{D}$ is assumed to be constant, reflection at the aortic valve can be chosen to be time-dependent [13], i.e. $R^{a v}=\left(R_{k}^{a v}\right)_{k \in \mathbb{N}}$ with the time index $k$ where the length of a time step will be given by the step size $\tau$. Typically, different values are chosen during systole, when the valve is open, and diastole, when the valve is closed.

The number of time steps it takes a pressure impulse to travel from the measuring site to the distal reflection site and back will be denoted by the time constant $t_{b} \in \mathbb{N}$. It includes information on both the distance between measuring site and reflection site and pulse wave velocity. In a more complex description of reflection phenomena the time of return of the reflected wave is also affected by the phase shift that occurs during re- flection [14]. Due to the simplification of the reflection coefficient in this paper this time delay must be included in the time constant. The choice of the time constant is coupled with the choice of the reflection coefficient $R_{D}$ $[14,15,16]$.

Analogously, the number of time steps it takes a pressure impulse to travel from the measuring site to the aortic valve, denoted by $t_{f} \in \mathbb{N}$, is coupled with the choice of $R^{a v}$. Since the measuring site is very close to the valve there holds $t_{f} \ll t_{b}$.

Not only does the heart serve as a reflection site, it also is the driving force for pressure dynamics. During systole blood is pumped into the system, increasing the pressure. This phenomenon is represented by the discrete function $\left(P_{k}^{i n}\right)_{k \in \mathbb{N}}$.

Model Equations and Solutions. In order to formulate the model equations total pressure $P_{k}$ (at the measuring site, at time $k$ ) is split into its forward and backward components $P_{k}^{f}$ and $P_{k}^{b}$, i.e. $P_{k}=P_{k}^{f}+P_{k}^{b}$. Using all the aforementioned parameters the difference equation system

$$
\begin{array}{ll}
P_{k}^{f}=P_{k}^{i n}+P_{k-2 t_{f}}^{b} R_{k-t_{f}}^{a v}, & k \in \mathbb{N}_{>2 t_{f},}, \\
P_{k}^{b}=P_{k-t_{b}}^{f} R_{D}, & k \in \mathbb{N}_{>t_{b}}
\end{array}
$$

can be obtained. Figure 2 illustrates the idea behind equation (1).

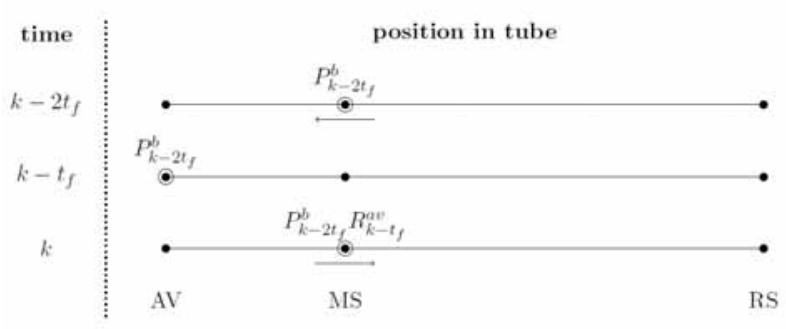

Figure 2: Backward pressure is reflected at the time-dependent aortic valve (AV) as described in equation (1) and returns to measuring site (MS).

Inserting (1) into (2) and vice versa yields the two independent equations

$$
\begin{array}{ll}
P_{k}^{f}=P_{k}^{i n}+P_{k-2 t_{f}-t_{b}}^{f} R_{D} R_{k-t_{f}}^{a v}, & k \in \mathbb{N}_{>2 t_{f}+t_{b},}, \\
P_{k}^{b}=P_{k-t_{b}}^{i n} R_{D}+P_{k-2 t_{f}-t_{b}}^{b} R_{D} R_{k-t_{b}-t_{f}}^{a v}, & k \in \mathbb{N}_{>2 t_{f}+t_{b} .}
\end{array}
$$

Obviously both equations are of order $N$ where $N=$ $2 t_{f}+t_{b}$. Since they only define a recurrence relation 
for $k>N$, initial values $P_{k}^{f}$ and $P_{k}^{b}, k=1, \ldots, N$ are required. Then standard literature [17] guarantees the existence of unique solutions for the resulting initial value problems. The initial values are chosen as $P_{k}^{f}=P_{k}^{i n}$, $k=1, \ldots, N, P_{k}^{b}=0, k=1, \ldots, t_{b}$ and $P_{k}^{b}=P_{k-t_{b}}^{i n} R_{D}$, $k=t_{b}+1, \ldots, N$. Using a solution method for linear difference equations of fixed but unknown order [18] yields the solutions

$P_{k}^{f}=P_{k}^{i n}+\sum_{j=1}^{\left\lfloor\frac{k-1}{2 t_{f}+t_{b}}\right\rfloor} P_{k-j\left(2 t_{f}+t_{b}\right)}^{i n} R_{D}^{j} \prod_{m=1}^{j} R_{k-(m-1)\left(2 t_{f}+t_{b}\right)-t_{f}}^{a v}$

and

$$
\begin{aligned}
P_{k}^{b}=P_{k-2 t_{b}}^{i n} R_{D}+\sum_{j=1}^{\left\lfloor\frac{k-t_{b}-1}{2 t_{f}+t_{b}}\right\rfloor}\left(P_{k-j\left(2 t_{f}+t_{b}\right)-t_{b}}^{i n} R_{D}^{j+1}\right. \\
\\
\left.\prod_{m=1}^{j} R_{k-(m-1)\left(2 t_{f}+t_{b}\right)-t_{f}-t_{b}}^{a v}\right)
\end{aligned}
$$

for $k \in \mathbb{N}_{>2 t_{f}+t_{b}}$.

Model Properties. Using stability theory for linear difference equation systems [17] it can be shown that all the solutions are stable and bounded under the assumption $R^{a v} \equiv 1$. For this purpose both independent equations of order $N$ have to be transformed to an equivalent difference equation system

$$
u_{k+1}=A u_{k}+b_{k+1}
$$

of order one with system matrix

$$
A=\left(\begin{array}{cccccc}
0 & 1 & 0 & \ldots & \ldots & 0 \\
\vdots & 0 & 1 & \ddots & & \vdots \\
\vdots & \vdots & \ddots & \ddots & \ddots & \vdots \\
\vdots & \vdots & & \ddots & \ddots & 0 \\
0 & \vdots & & & \ddots & 1 \\
R_{D} & 0 & \ldots & \ldots & \ldots & 0
\end{array}\right) \quad \in \mathbb{R}^{N \times N}
$$

Then, for any matrix norm $\|\cdot\|$ there holds

$$
\lim _{k \rightarrow \infty}\left\|A^{k}\right\|=0 .
$$

Let $\tilde{A}$ be the system matrix of the equivalent difference equation system of order one with variable $R^{a v}$. Be- cause of

$$
\left\|\tilde{A}^{k}\right\| \leq\left\|A^{k}\right\| .
$$

for any matrix norm $\|\cdot\|$ the same result is valid for this system.

Both functions $\left(R_{k}^{a v}\right)_{k \in \mathbb{N}}$ and $\left(P_{k}^{i n}\right)_{k \in \mathbb{N}}$ are periodic with the same period length $K$ that equals the duration of one cardiac cycle, i.e. the sum of systole and diastole duration. Therefore, also the solutions of the model become periodic with period length $K$ after a certain setting time that depends on the choice of the reflection coefficient $R_{D}$.

Under the assumptions made for the model it is possible to set $t_{f}=0$, i.e. shift the measuring site towards the heart, without major changes in the results. The solution then simplifies to

$P_{k}=P_{k}^{f}+P_{k}^{b}=P_{k}^{i n}+\sum_{j=1}^{\left\lfloor\frac{k-1}{t_{b}}\right\rfloor} P_{k-j t_{b}}^{i n} R_{D}^{j}\left(R_{k}^{a v}+1\right) \prod_{m=1}^{j-1} R_{k-m t_{b}}^{a v}$

for $k \in \mathbb{N}_{>2 t_{f}+t_{b}}$. In order to simplify analysis this will be done in the rest of the paper.

\section{Implementation and Results}

After theoretical discussion the model is implemented in MATLAB. Numerical analysis is conducted by comparing plots of generated curves where one parameter is varied while the others are held constant. Both the influence of parameters on the shape of the curve and on the minimum (diastolic pressure) and maximum (systolic pressure) values are analyzed.

Parameter Values and Implementation. Parameter ranges are determined according to previous studies. Systole duration typically is 0.3 seconds, and diastole duration typically is 0.7 [19]. They are included in the model via the choice of the input function $P^{i n}$ and the reflection at the heart $R^{a v}$.

The distal reflection coefficient should be chosen around $R_{D}=0.5$. Different authors give different values that are computed using different methods $[14,16,20]$.

The same goes for the return time from the distal reflection site where both effective length and pulse wave velocity can be determined in various ways $[14,16,21]$. A typical value is 145 milliseconds which is much shorter than systole duration [22]. This time constant can be transformed to a discrete number of time steps 
using the number of time points per second $1 / \tau$. Implementation of the model using the previously defined parameters and initial values in MATLAB is straightforward.

Input Function and Reflection at the Heart. Two different functions are compared for $P^{i n}$. Both functions are zero during diastole when the valve is closed and the heart produces no output. During systole a half sine that is motivated by other authors' results $[2,13]$ is compared to the much simpler constant one function. The half sine (see Figure 3 ) that considers the opening and closing time of the aortic valve yields more realistic results than the strongly simplified constant function (see Figure4).

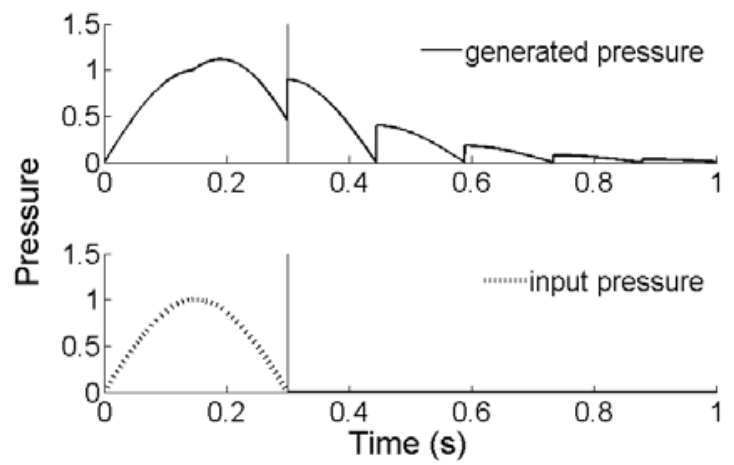

Figure 3: Generated pressure curve (solid) with corresponding input function (dotted). A half sine is chosen during systole. Reflection at the heart is zero during systole and one during diastole. End of systole is indicated by a vertical line. One cardiac cycle.

For modeling reflection at the heart three different functions can be compared. A step function assumes zero during systole when the valve is open and all waves are absorbed and one during diastole when the valve is closed and all waves are reflected. As before, motivation for this function is given by other authors [13]. Instead, the simpler constant functions zero and one can be used. They produce smoother curves with less distinct spikes, see Figure 5. In the following analysis the reflection is assumed to be a step function.

Results. Figure 6 compares a curve generated by the model (solid) with a measured blood pressure curve (dotted) taken from a data set that was collected for a previous study [23]. Both curves are normalized to the

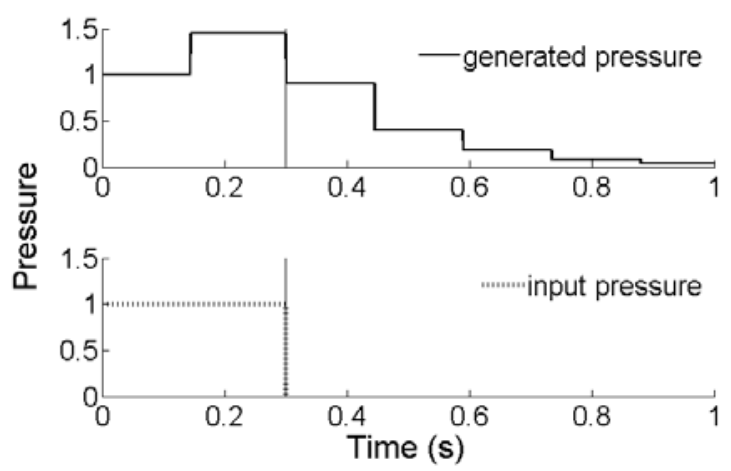

Figure 4: Generated pressure curve (solid) with corresponding input function (dotted). The constant one function is chosen during systole. Reflection at the heart is zero during systole and one during diastole. End of systole is indicated by a vertical line. One cardiac cycle.

same pulse pressure (amplitude) to increase comparability. The focus of this paper is the shape of blood pressure curves, not their absolute values. For the generated curve the input function $P^{\text {in }}$ is chosen as a half sine during systole and zero during diastole. Reflection at the heart, $R^{a v}$, is chosen as the constant one function during systole and zero during diastole. A vertical line indicates the end of systole.

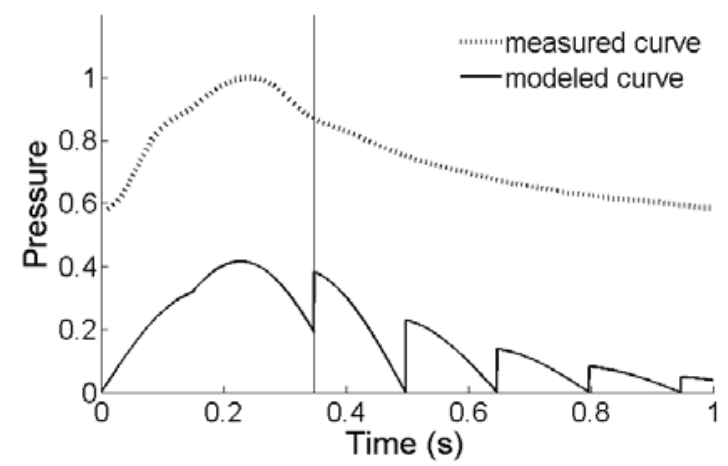

Figure 6: Comparison of a modeled (solid) and a measured (dotted) blood pressure curve. End of systole is indicated by a vertical line. One cardiac cycle.

Even though the modeled curve shows more spikes and is generally less smooth, the main characteristics of an aortic blood pressure curve [24] are represented. There is a (relatively) fast systolic upstroke, a notch at the end of systole and a slow diastolic decay.

Increasing the reflection coefficient $R_{D}$ significantly 


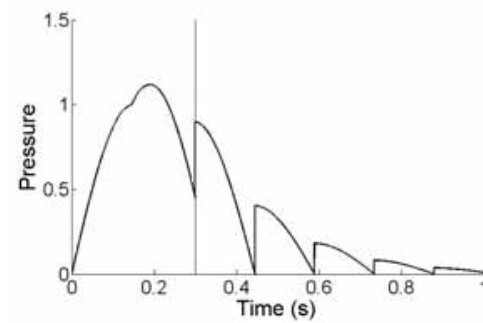

(a) $R^{a v}$ is step function.

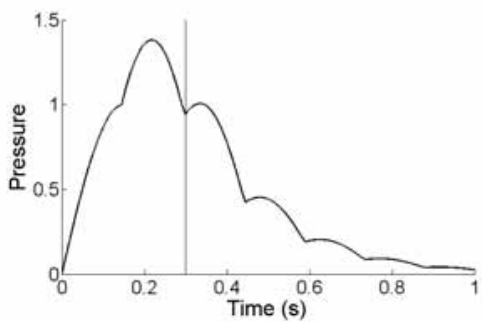

(b) $R^{a v}$ is constant one function.

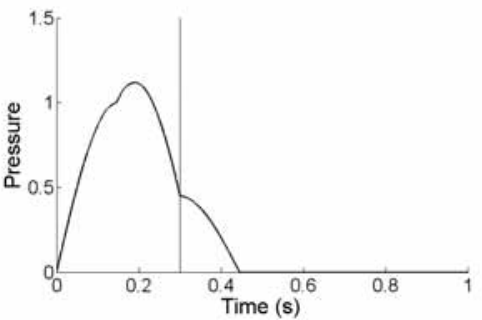

(c) $R^{a v}$ is constant zero function.

Figure 5: Comparison of different shapes of $R^{a v}$. Input function is a half sine during systole and zero during diastole. End of systole is indicated by a vertical line. One cardiac cycle.

increases systolic pressure and the prominence of spikes while diastolic pressure remains close to zero, yielding an increase in pulse pressure, i.e. the amplitude of the wave. The curves look realistic only for a sufficiently small value of $R_{D}$ where the threshold depends on the choice of other parameters.

In assessing the influence of systole and diastole duration one must distinguish between two cases. One can either vary them individually, resulting in a variable heart rate or hold the heart rate fixed which means varying them together. Only the first case that is physiologically more relevant is analyzed in the paper. Systolic pressure increases with systole duration while diastolic pressure remains the same, yielding an increase in pulse pressure, see Figure 7. The prominence of spikes decreases. Both for too low and too high values of systole duration the curve does not resemble a measured one. The thresholds depend on the values of the other parameters. Diastole duration does not have a significant influence on the shape of the modeled curves.

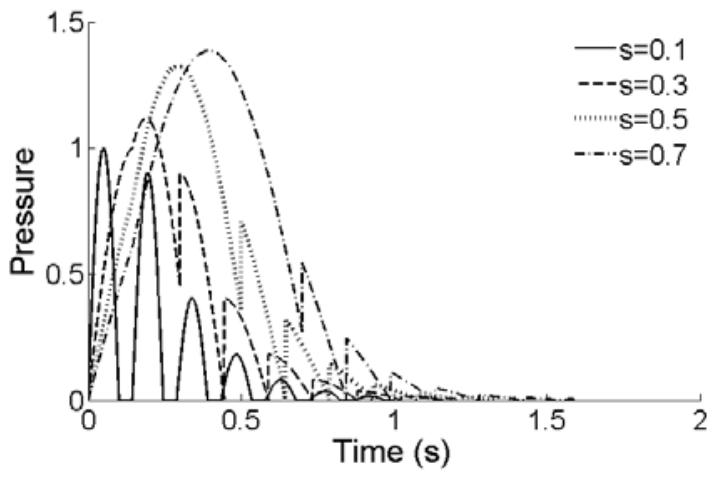

Figure 7: Generated blood pressure curve for four different values of systole duration. One cardiac cycle.

Return time $t_{b}$ negatively influences systolic and pulse pressure while diastolic pressure remains zero. All spikes occurring during diastole become wider and more prominent with increasing return time, see Figures 8 and 9 . The width of spikes during diastole equals the return time, therefore representing the single reflections.

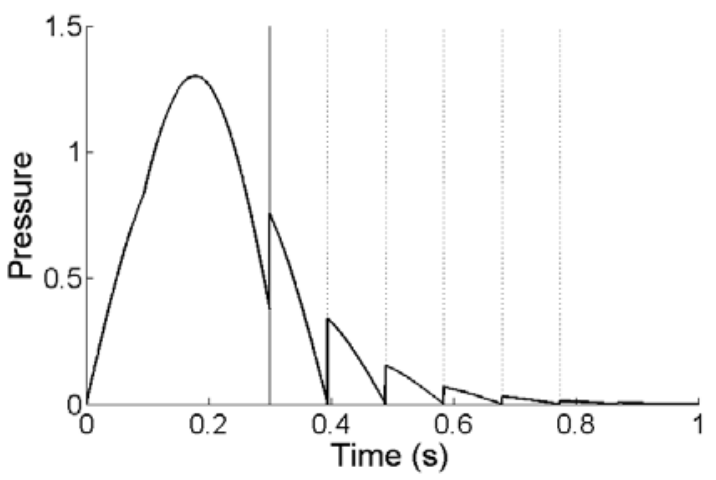

Figure 8: Return time 95ms. End of systole is indicated by a solid vertical line. Return times are indicated by dotted vertical lines. One cardiac cycle.

Choosing a return time that is larger than systole duration does not lead to realistic results.

\section{Model Refinement}

The curves' shapes can be improved by adding more reflection sites. It has been argued before that at least two peripheral reflection sites are necessary to model the entire arterial system - one for the upper and one for the lower body half $[25,26]$.

The Model. While modeling two reflection sites is often accomplished with an asymmetric T-tube model 


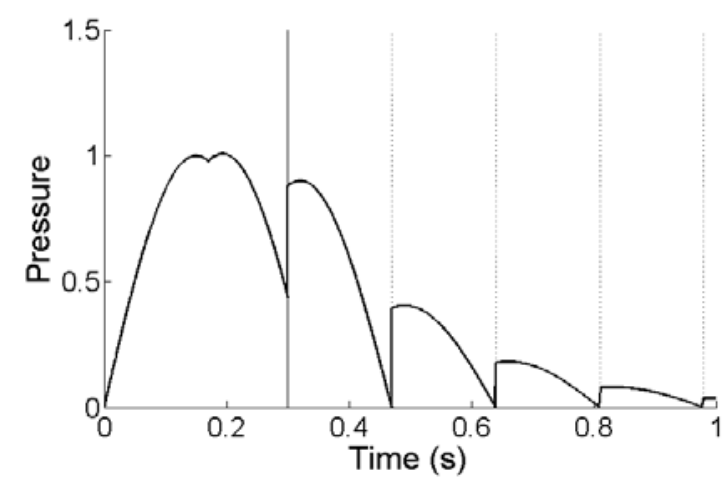

Figure 9: Return time 170ms. End of systole is indicated by a solid vertical line. Return times are indicated by dotted vertical lines. One cardiac cycle.

[27], the reflection sites in the present paper are assumed to be completely independent from each other. The equations are

$$
\begin{array}{ll}
P_{k}^{f}=P_{k}^{i n}+P_{k-2 t_{f}}^{b} R_{k-t_{f}}^{a v}, & k \in \mathbb{N}_{>2 t_{f},}, \\
P_{k}^{b}=P_{k-t_{b}^{1}}^{f} R_{D}^{1}+P_{k-t_{b}^{2}}^{f} R_{D}^{2}, & k \in \mathbb{N}_{>t_{b}^{2}}
\end{array}
$$

where $R_{D}^{1}$ and $R_{D}^{2}$ are the reflection coefficients of the two reflection sites. Their respective return times are $t_{b}^{1}$ and $t_{b}^{2}$ with $t_{b}^{1}<t_{b}^{2}$.

Inserting each of the equations into the other one and prescribing initial values $P_{k}^{f}$ and $P_{k}^{b}$ for $k=1, \ldots, N=$ $2 t_{f}+t_{b}^{2}$ yields two initial value problems. Standard literature [17] guarantees the existence of unique solutions. They cannot be expressed explicitly. It is possible, however, to prove analytically that the solutions of the homogeneous problems are bounded as long as $R_{D}^{1}+R_{D}^{2}<1$. In the following, $t_{f}=0$ will be assumed.

Results. Comparison with the original model reveals that the prominence of spikes during diastole is decreased significantly, see Figure 10. In particular, blood pressure does not decrease to zero after each spike, presumably a result of the superposition of reflected waves. The main characteristics of a blood pressure curve are represented.

It can be observed that the generated curves are both bounded and periodic after a certain setting time, even for $R_{D}^{1}+R_{D}^{2} \geq 1$.

Again, the influence of different parameters can be assessed. While the general effects are the same as in the model with only one reflection site, the different in-

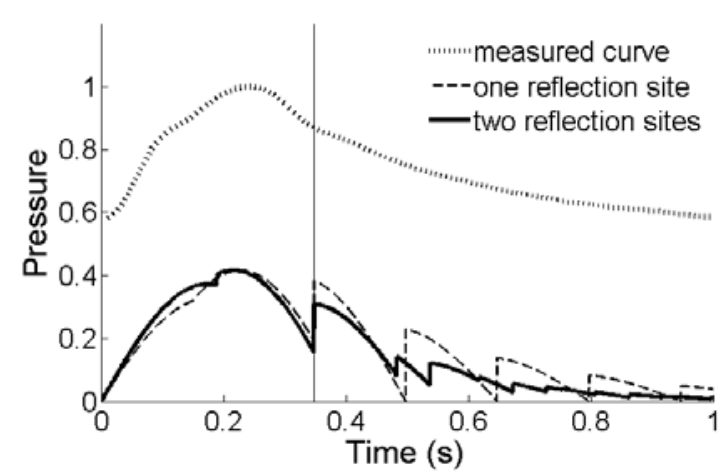

Figure 10: Comparison of a measured curve (dotted), a curve modeled with one reflection site (dashed) and a curve modeled with two reflection sites (solid). End of systole is indicated by a vertical line. One cardiac cycle.

fluences of the two reflection sites can be compared. The reflection coefficients have similar influences on systolic and diastolic pressure as long as both are small. When they exceed a certain threshold that depends on the choice of the other parameters, $R_{D}^{1}$ increases systolic blood pressure and $R_{D}^{2}$ increases diastolic blood pressure more significantly. Physiologically meaningful results can only be obtained when the sum $R_{D}^{1}+R_{D}^{2}$ does not exceed some threshold value that depends on the return times. Each of the reflection coefficients has to be chosen smaller than the reflection coefficient in the original model.

The influence of systole and diastole duration is the same as can be observed in the original model with one reflection site. The same is valid for the influence of both return times.

\section{Conclusion and Outlook}

All the results obtained by theoretical and numerical analysis correspond to effects observed in reality and therefore indicate that modeling aortic blood pressure with difference equations is a reasonable approach.

Instead of a variable reflection at the heart constant functions can be chosen. If the heart is considered to be a total reflector during the entire cardiac cycle, or equivalently, $R^{a v} \equiv 1$, estimating formulas for systolic and diastolic pressure can be found, enabling further theoretical analysis. In fact, choosing $R^{a v}$ close to 1 is necessary 
if physiologically meaningful absolute values, i.e.

diastolic pressure : pulse pressure $\approx 2: 1$,

are desired. This indicates that re-reflections at the aortic valve should not be neglected in methods of arterial pulse wave analysis.

The model can be further refined by adding more distal reflection sites. Also, more complex functions to model reflections at the heart can be chosen. These could be step functions but also piecewise linear functions that consider opening and closing of the valve. Another possibility is to find a model to describes the ventricle. During systole this model is linked with the arterial system while during diastole reflection is assumed to be total.

Once the model has been improved to a desired level of exactness, it can be fit to measured curves by determining the correct parameter combination. From this point, a variety of applications can be considered, such as implementing a wave separation [6] or wave tracking [28] algorithm.

\section{References}

[1] Mendis S, Puska P, Norrving B, editors. Global Atlas on cardiovascular disease prevention and control. World Health Organization, 2011.

[2] Formaggia L, Lamponi D, Quarteroni A. One-dimensional models for blood flow in arteries. Journal of Engineering Mathematics. 2003; 47:251-276. doi: 10.1023/B:ENGI.0000007980.01347.29.

[3] Formaggia L, Perktold K, Quarteroni A. Basic mathematical models and motivations. In: Formaggia, L., Quarteroni, A. and Veneziani, A., editors. Cardiovascular Mathematics. Milano: Springer; 2009. p 47-75.

[4] Westerhof N, Lankhaar JW, Westerhof BE. The arterial Windkessel. Medical and Biological Engineering and Computing. 2009; 47:131-141. doi: 10.1007/s11517-008-0359-2.

[5] Papageorgiou GL, Jones NB. Wave reflection and hydraulic impedance in the healthy arterial system: a controversial subject. Medical and Biological Engineering and Computing. 1988; 26:237-242. doi: 10.1007/BF02447075.
[6] Westerhof N, Sipkema P, van den Bos GC, Elzinga G. Forward and backward waves in the arterial system. Cardiovascular Research. 1972; 6:648-656. doi: 10.1093/cvr/6.6.648.

[7] Berger DS, Li JKJ, Laskey WK, Noordergraaf A. Repeated reflection of waves in the system arterial system. American Journal of Physiology - Heart and Circulatory Physiology. 1993; 264:H269-H281. doi: 10.1152/ajpheart.1993.264.1.H269.

[8] Westerhof N, Stergiopulos N, Noble MIM. Snapshots of Hemodynamics. Boston: Springer; 2005. 192 p.

[9] Burattini R, Knowlen GG, Campbell KB. Two arterial effective reflecting sites may appear as one to the heart. Circulation Research. 1991; 68: 85-99. doi: 10.1161/01.RES.68.1.85.

[10] Caro CG, Pedley TC, Schroter RC, Seed WA. The Mechanics of the Circulation. New York: Cambridge University Press; 2012. 550 p.

[11] Womersley JR. Oscillatory flow in arteries: the constrained elastic tube as a model of arterial flow and pulse transmission. Physics in Medicine and Biology. 1957; 2:178-187. doi: 10.1088/0031-9155/2/2/305.

[12] Nichols WW, O’Rourke MF, Vlachopoulos C. McDonald's Blood Flow in Arteries, Sixth Edition: Theoretical, Experimental and Clinical Principles. London: CRC Press; 2011. 768p.

[13] Wang JJ, Parker KH. Wave propagation in a model of the arterial circulation. Journal of Biomechanics. 2004; 37:457-470. doi: 10.1016/j.jbiomech.2003.09.007.

[14] Westerhof BE, van den Wijngaard JP, Murgo JP, Westerhof N. Location of a reflection site is elusive. Hypertension. 2008; 52:478-483. doi: 10.1161/HYPERTENSIONAHA.108.116525.

[15] Campbell KB, Lee LC, Frasch HF, Noordergraaf A. Pulse reflection site and effective length of the arterial system. American Journal of Physiology - Heart and Circulatory Physiology. 1989; 256:H1684-H1689. doi: 10.1152/ajpheart.1989.256.6.H1684.

[16] Segers P, De Backer J, Devos D, et al. Aortic reflection coefficients and their association with global indexes of wave reflection in healthy controls and patients with Marfan's syndrome. American Journal of Physiology - Heart and Circulatory Physiology. 2006; 290(6):H2385-H2392. doi: 10.1152/ajpheart.01207.2005. 
[17] Agarwal RP. Difference Equations and Inequalities. New York: Marcel Dekker Inc; 1992. 1000 p.

[18] Mallik RK. Solutions of linear difference equations with variable coefficients. Journal of Mathematical Analysis and Applications. 1998; 222:79-91. doi: 10.1006/jmaa.1997.5903.

[19] Thiriet M, Parker KH. Physiology and pathology of the cardiovascular system: a physical perspective. In: Formaggia, L., Quarteroni, A. and Veneziani, A., editors. Cardiovascular Mathematics. Milano: Springer; 2009. p 1-45.

[20] Latham RD, Westerhof N, Giolma JP, Altobelli SA. Regional wave travel and reflections along the human aorta: a study with six simultaneous micromanometric pressures. Circulation. 1985; 72:1257-1269. doi: 10.1161/01.CIR.72.6.1257.

[21] Murgo JP, Westerhof N, Giolma JP, et al. Aortic input impedance in normal man: Relationship to pressure wave forms. Circulation. 1980; 62: 105-116. doi: 10.1161/01.CIR.62.1.105

[22] Sherwin SJ, Franke V, Peiro J, Parker KH. One-dimensional modelling of a vascular network in space-time variables. Journal of Engineering Mathematics. 2003; 47:217-250. doi: 10.1023/B:ENGI.0000007979.32871.e2.
[23] Weber T, Auer J, O’Rourke MF, et al. Arterial Stiffness, wave reflections, and the risk of coronary artery disease. Circulation. 2004; 109:184-189. doi: 10.1161/01.CIR.0000105767.94169.E3.

[24] Stoner L, Young JM, Fryer S. Assessments of arterial stiffness and endothelial function using pulse wave analysis. International Journal of Vascular Medicine. 2012; Article ID 903107.. doi: 10.1155/2012/903107.

[25] O'Rourke MF, Taylor MG. Input Impedance of the System Circulation. Circulation Research. 1967; 20:365-380. doi: 10.1161/01.RES.20.4.365.

[26] Sipkema P, Westerhof N, Randall OS. The arterial system characterised in the time domain. Cardiovascular Research. 1980; 14:270-279. doi: 10.1093/cvr/14.5.270.

[27] O'Rourke MF. The arterial pulse in health and disease. American Heart Journal. 1971; 82:687-702. doi:10.1016/0002-8703(71)90340-1.

[28] Parker KH. The reservoir-wave model. Artery Research. 2017; 18:87-101. doi:10.1016/j.artres.2017.04.003. 